\title{
Review: statins reduce non-fatal and fatal strokes in patients with and without coronary heart disease
}

Briel M, Studer M, Glass TR, et al. Effects of statins on stroke prevention in patients with and without coronary heart disease: a metaanalysis of randomized controlled trials. Am J Med 2004;117:596-606.

In patients with and without coronary heart disease (CHD), what are the effects of lipid lowering interventions (statins, fibrates, resins, n-3 fatty acids, and diet) for preventing non-fatal and fatal strokes?

\section{METHODS}

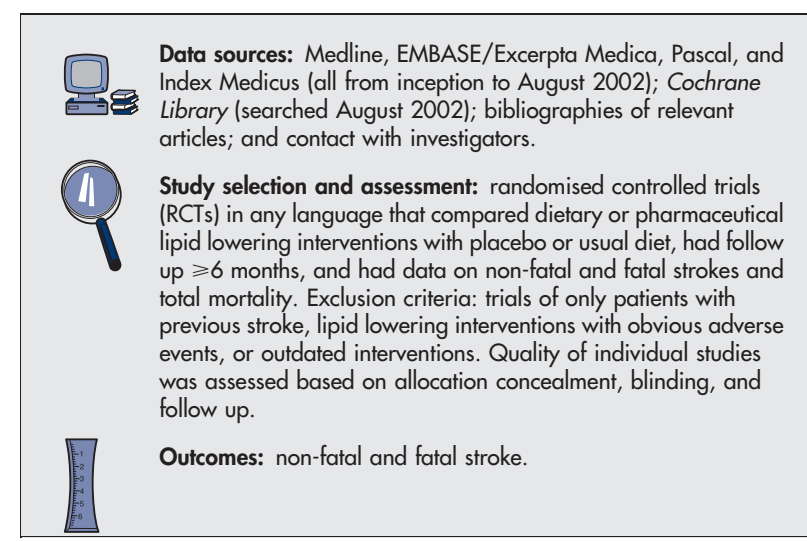

\section{MAIN RESULTS}

65 RCTs were included $(\mathrm{n}=97502$ for the pooled intervention group and $n=103105$ for the pooled control group). Statins reduced the risk of non-fatal and fatal stroke more than control interventions in patients with and without CHD combined, and in subgroups of patients with or without CHD (table). Fibrates, resins, n-3 fatty acids, and dietary interventions were not more effective than control interventions for reducing risk of non-fatal and fatal stroke.

\section{CONCLUSION}

In patients with and without coronary heart disease, statins reduce non-fatal and fatal stroke, whereas fibrates, resins, n-3 fatty acids, and dietary interventions have no such effect.
For correspondence: $\operatorname{Dr} M$ Briel, Basel Institute for Clinical Epidemiology University Hospital Basel, Basel, Switzerland. brielm@uhbs.ch Source of funding: no external funding.

\section{Commentary}

troke is the third leading cause of death in industrialised countries, preceded by cardiovascular disease and cancer. ${ }^{1}$ Management of lipids is considered to be an important component of stroke prevention, although the benefits of lipid lowering for stroke prevention have not been fully elucidated. Another meta-analysis ${ }^{2}$ found a $17 \%$ relative risk reduction (RRR) of stroke with lipid lowering treatment (no heterogeneity among trials or among subgroups categorised by primary versus secondary prevention or type of lipid lowering treatment). Statins had the most substantial effects (RRR 26\%). It is important to note that fatal and non-fatal strokes were not primary endpoints in the trials included in this meta-analysis, and only between-study comparisons were made. Participants in statin trials were older and had higher rates of fatal and non-fatal stroke.

The findings of the review by Briel et al underscore the importance of statin treatment to reduce the incidence of stroke in patients with and without heart disease. As a drug class, statins are thought to have numerous pleiotropic effects. These are statin effects not achieved through lipoprotein changes, including anti-inflammatory and endothelial protective effects that are independent of lipid lowering, thus conferring additional protection against vascular events. ${ }^{3}$ Although the authors did not confirm the benefits of non-statin lipid lowering treatment, the merits of such treatment should not be dismissed. The finding of a lack of treatment effect with non-statin treatment could be the result of small cohorts or confounding by between-study comparisons. Despite the known benefits of statins, many patients fail to receive adequate treatment. Nurses have an important role in the assessment and treatment of cardiovascular and stroke risk factors and management of pharmaceutical interventions. The optimal intervention to mitigate stroke risk in patients with and without heart disease should include efforts aimed at reducing conventional risk factors and the initiation of statin treatment.

Kori J Kingsbury, RN, MSN St Paul's Hospital

Vancouver, British Columbia, Canada

1 Hsu CY, editor. Ischemic stroke: from basic mechanisms to new drug development. (Monographs in Clinical Neuroscience, Volume 16.) Karger: Basel, Switzerland, 1998.

2 Corvol JC, Bouzamondo A, Sirol M, et al. Differential effects of lipidlowering therapies on stroke prevention: a meta-analysis of randomized trials. Arch Intern Med 2003;163:669-76.

3 Ky B, Rader DJ. The effects of statin therapy on plasma markers of inflammation in patients without vascular disease. Clin Cardiol 2005;28:67-70

Statins $v$ control interventions for prevention of non-fatal and fatal stroke (follow up 0.5 to $6.1 \mathrm{y}$ )

\begin{tabular}{llllll}
\hline & & \multicolumn{2}{l}{ Weighted event rates } & & \\
\cline { 3 - 4 } Patients & Number of trials $(\mathbf{n})$ & Statins & Control & RRR (95\% Cl) & NNT (CI) \\
\hline With and without CHD & $29(93399)$ & $3.2 \%$ & $3.6 \%$ & $18 \%(10$ to 24$)$ & 220 (140 to 504$)$ \\
With CHD & $17(25718)$ & $2.6 \%$ & $3.2 \%$ & $25 \%(13$ to 35$)$ & 190 (112 to 634) \\
Without CHD & $6(19567)$ & $1.4 \%$ & $1.9 \%$ & $23 \%$ (5 to 38) & 159 (129 to 1667)
\end{tabular}

${ }^{*} \mathrm{CHD}=$ coronary heart disease; other abbreviations defined in glossary. RRR, NNT, and Cl calculated from data in article using a random effects model. Patients in 6 of the 29 statin trials could not be classified as having or not having CHD because 10-90\% of patients had CHD. 